\title{
ASYMPTOTIC CONVERGENCE OF TRAJECTORIES OF QUADRATIC DIFFERENTIALS
}

\author{
ALBERT MARDEN ${ }^{11}$ and KURT STREBEL
}

1. Introduction. In this article we continue our investigations of the geometric properties of holomorphic quadratic differentials. We will consider only the case of a compact Riemann surface $R$, leaving for another time the extension to the more general situation that $R$ is allowed a finite number of punctures.

The first part is an investigation of the convergence of trajectory rays, or more generally, geodesic rays, induced by the covergence of the differentials. The phenomenon can be studied on the surface itself or - and this is our objective - in terms of the lifts to the universal covering surface $\boldsymbol{H}$ represented by the unit disk.

Let $\left\{\varphi_{n}\right\}$ be a sequence of holomorphic quadratic differentials on $R$ which converges locally uniformly to a differential $\varphi$. In view of the compactness of $R$, the locally uniform convergence is equivalent to the ccnvergence in norm $\left\|\varphi_{n}-\varphi\right\| \rightarrow 0$. Since the case $\varphi \equiv 0$ is of no interest and the trajectory structure of a quadratic differential is independent of a positive constant factor we may normalize all differentials so that

$$
\left\|\varphi_{n}\right\|=\|\varphi\|=\iint|\varphi| d u d v=1 .
$$

We will denote the space of normalized differentials by $Q(R)$.

A (horizontal) trajectory ray $\alpha$ of $\varphi$ is a maximal arc, starting at some point $p \in R$ along which, in terms of any local parameter $w, \varphi(w) d w^{2}>0$. It is called critical if it ends at a zero of $\varphi$; otherwise it is called regular. A critical ray can be continued across the zero $\zeta$ as a geodesic ray. It must then satisfy Teichmüller's angle condition at $\zeta$

$$
\vartheta \geqq 2 \pi /(k+2)
$$

but otherwise, the direction of continuation is free: it is not necessarily horizontal. We always think of a geodesic ray as continued indefinitely. It can have self crossings on the surface, whereas a regular ray cannot meet itself, unless it closes up forming on $R$ a simple loop.

A geodesic is the union of a finite or countably infinite number of $\varphi$-straight segments or rays (along each of which $\arg \left(\varphi(w) d w^{2}\right)$ is constant) whose endpoints are zeros of $\varphi$. A geodesic is called horizontal if $\varphi d z^{2}>0$ along each regular part.

1) Supported in part by the National Science Foundation. 
The critical graph of $\varphi$ is the union of all zeros of $\varphi$ and the (horizontal) trajectory rays emanating from them.

A quadratic differential is said to have closed trajectories if all of its (non-critical) trajectories are closed. This is the case, on a compact surface, if and only if the critical graph is compact. The closed trajectories sweep out disjoint annuli. A quadratic differential with closed trajectories is called simple if all of its closed trajectories are parallel (sweep out a single annulus).

An approximation theorem due to Masur [4] says that any quadratic differential can be approximated by simple ones. In the sense of Thurston (see e.g. [2]) the closed trajectories of the approximating $\varphi_{n}$ converge. It is the motivation for our work to study the asymptotic convergence of these trajectories.

The quadratic differentials are lifted to the universal covering surface $\boldsymbol{H}$ by means of the holomorphic projection map $\pi: H \rightarrow R$,

$$
\varphi^{*}(z) d z^{2}=\varphi(\pi(z)) \pi^{\prime}(z)^{2} d z^{2} .
$$

If $\alpha$ is a trajectory of $\varphi$ in $R$ then each component of $\left\{\pi^{-1}(\alpha)\right\}$ in $\boldsymbol{H}$ is a trajectory of $\varphi^{*}$ which, as shown in [3], has distinct end points on the circle $\partial \boldsymbol{H}$. Conversely each trajectory of $\varphi^{*}$ projects to one of $\varphi$. More generally if $\alpha$ is a geodesic of $\varphi$ and $w \in \alpha$ is a regular point, through each point $w^{*}$ over $w$ passes a lift $\alpha^{*}$ of $\alpha$. This lift $\alpha^{*}$ is a geodesic for $\varphi^{*}$ and it is a simple arc in $\boldsymbol{H}$ which [3] has distinct end points on $\partial \boldsymbol{H}$. And conversely, the projection to $R$ of a geodesic for $\varphi^{*}$ is a geodesic for $\varphi$ (but not necessarily simple).

If $f: R \rightarrow S$ is a homeomorphism between two Riemann surfaces, $f$ induces both a) a homeomorphism $f_{\#}: Q(R) \rightarrow Q(S)$ between the $(6 g-7)$-dimensional spheres of normalized differentials and b) a homeomorphism $f^{*}: \partial \boldsymbol{H} \rightarrow \partial \boldsymbol{H}$ of the unit circle. Both $f_{\#}$ and $f^{*}$ depend only on the homotopy class of $f$. The mapping $f_{\#}$ was constructed in [2], and the existence of $f^{*}$ is a classical result of Nielsen. In the context of analysis the existence of $f^{*}: \partial \boldsymbol{H} \rightarrow \partial \boldsymbol{H}$ results from taking $f$ to be quasiconformal, lifting $f$ to a necessarily quasiconformal map $f^{*}: \boldsymbol{H} \rightarrow \boldsymbol{H}$, and extending that. As a map of the circle, $f^{*}$ is uniquely determined by the homotopy class of $f$ up to a composition $T_{2} \circ f^{*} \circ T_{1}$ where $T_{1}$ is a cover transformation over $R$ and $T_{2}$ is one over $S$.

The second part of our work, which depends heavily on the first, shows how the maps $f_{\#}$ and $f^{*}$ are tied together. For a generic differential $\varphi \in Q(R)$ we show in particular that points $p, q \in \partial \boldsymbol{H}$ are the end points of a trajectory of its lift $\varphi^{*}$ in $\boldsymbol{H}$ if and only if $f^{*}(p), f^{*}(q)$ are the end points of a trajectory of the lift of $f_{\#}(\varphi)$. This is our main result.

2. We refer to the result, established in Marden and Strebel [3], that a lift $\gamma^{*}$ of any geodesic ray $\gamma$ of $\varphi$ (i.e. any geodesic ray of the lift $\varphi^{*}$ of $\varphi$ ) has a well determined end point on $\partial \boldsymbol{H}$. In order to show the convergence of the rays, we need however more information about the associated metric. The main difficulty lies in the 
fact that the lifts $\varphi^{*}$ have infinite norm and the convergence, in $\boldsymbol{H}$, is only locally uniform.

Lemma 1 (Annulus lemma). Let $A$ be a circular annulus and $\sigma$ a simple loop separating its boundary contours. Suppose $\Psi$ is an arbitrary holomorphic differential in $A$ and $\gamma$ is a simple geodesic segment of $\Psi$ whose end points lie on $\sigma$ but which has no other points in common with $\sigma$. Then, for the $\Psi$-lengths,

$$
|\gamma|_{\Psi} \leqq|\sigma|_{\Psi} .
$$

Proof: Assume, first, that the arc $\gamma$ has two different end points. Then, it bounds a simply connected region $D$ together with one of the subarcs $\sigma_{1}$ of $\sigma$ determined by the end points of $\gamma$. Because $\gamma$ is the unique $\Psi$-geodesic in its homotopy class,

$$
|\gamma|_{\Psi} \leqq\left|\sigma_{1}\right|_{\Psi}<|\sigma|_{\Psi} .
$$

If the end points of $\gamma$ coincide, it is a Jordan curve which must contain the interior boundary of $A$. Otherwise it would contradict the uniqueness of geodesic connections in a simply connected domain. We can therefore apply the earlier argument, using $\sigma$ instead of $\sigma_{1}$. This proves the lemma.

The arc $\gamma$ is automatically simple, if it is a subarc of a trajectory, or any straight arc. This is the case in our application of the Annulus lemma.

The lemma allows for a sharper bound of the length of certain horizontal arcs in the following case.

Let $T$ be a hyperbolic or parabolic element of the group $G$ of cover transformations of $\boldsymbol{H}$. Let $\sigma^{*}$ be a circle in $\boldsymbol{H}$ along which the points move under $T$ (circle through the fixed point(s) of $T$ ). We form the corresponding annular covering surface $A$ by identifying the points of $\boldsymbol{H}$ equivalent under $\left\{T^{n}\right\}$. Suppose $\varphi^{*}$ is the lift to $\boldsymbol{H}$ of a holomorphic quadratic differential $\varphi$ on $R$, and $\Psi$ is the projection of $\varphi^{*}$ to $A$. The closed curve $\sigma$ is the projection of a fundamental subinterval $\sigma_{0}^{*}$ of $\sigma^{*}$ under $T$. Let $\alpha^{*}$ be a horizontal arc of $\varphi^{*}$ having its two end points on $\sigma^{*}$, but not meeting $\sigma^{*}$ otherwise. Then,

$$
\left|\alpha^{*}\right|_{\varphi^{*}} \leqq\left|\sigma_{0}^{*}\right|_{\varphi^{*}}
$$

A crude estimate would only compare the length of $\alpha^{*}$ with that of the subarc of $\sigma^{*}$ between its two end points. The lemma is actually of a topological nature.

3. In this section we show that $\boldsymbol{H}$ is uniformly complete in the $\varphi^{*}$-metrics, $\varphi \in Q(R)$ and in Section 4 we draw a number of conclusions from this fact.

Lemma 2 (Completeness). (i) For fixed $z_{0} \in \boldsymbol{H}$, given any $L>0$, there exists $r<1$ such that for any $z \in \boldsymbol{H}$ with $|z|>r$, and any path $\tau$ in $\boldsymbol{H}$ from $z_{0}$ to $z$, the $\varphi^{*-}$ length of $\tau$ exceeds $L$ for all $\varphi \in Q(R)$.

(ii) For fixed $\zeta^{\prime} \neq \zeta \in \partial \boldsymbol{H}$, given any $L>0$, there exists $\delta>0$ such that for any $z, z^{\prime} \in \boldsymbol{H}$ which satisfy $|\zeta-z|<\delta,\left|\zeta^{\prime}-z^{\prime}\right|<\delta$ and any path $\tau$ in $\boldsymbol{H}$ from $z$ to $z^{\prime}$, the $\varphi^{*}$ length of $\tau$ exceeds $L$ for all $\varphi \in Q(R)$. 
Proof: Cover $R$ with a finite system $\left\{U_{i}\right\}$ of simply connected neighborhoods such that for each $i$, the closure $U_{i}^{-}$is contained in a simply connected neighborhood $V_{i}$ bounded by a simple loop $\partial V_{i}$. Let $\tau$ be any arc in $\boldsymbol{H}$ from a point $z_{0}$ and which has limit points on $\partial \boldsymbol{H}$. We claim that there are infinitely many disjoint open intervals $\left\{\tau_{j}\right\}$ of $\tau$ such that for each $j$ there is some $i$ for which the closed arc $\pi\left(\tau_{j}^{-}\right)$runs in $V_{i}^{-} \backslash U_{i}$ from $\partial U_{i}$ to $\partial V_{i}$.

To prove this assertion we first dispose of the case that the projection $\pi(\tau)$ is a closed curve in $R$. The base point $\pi\left(z_{0}\right)$ lies in $U_{1}$, say. Since $\pi(\tau)$ cannot entirely lie in $V_{1}$ it contains an $\operatorname{arc} P$ from a point of $\partial U_{1}$ to a point of $\partial V_{1}$. The required arcs $\left\{\tau_{j}\right\}$ can be chosen from among the components of $\left\{\pi^{-1}(p)\right\}$.

If $\pi(\tau)$ is not closed we can find a sequence of points $z_{n} \in \tau$ with $\lim z_{n} \in \partial \boldsymbol{H}$ such that $\left\{\pi\left(z_{n}\right)\right\}$ are distinct points in $R$ with $\lim \pi\left(z_{n}\right)=p$. Assume say $p \in U_{1}$. Let $\varrho_{n}$ denote the interval of $\tau$ from $z_{n}$ to $z_{n+1}$. Its projection $\pi\left(\varrho_{n}\right)$ cannot lie in $V_{1}$ for all $n$ exceeding some number $N$. For if that happened then the entire part of $\tau$ beyond $z_{N+1}$ would lie in a component of $\left\{\pi^{-1}\left(V_{1}\right)\right\}$. Thus for an infinite number of indices $n, \pi\left(\varrho_{n}\right)$ contains a segment running in $V_{1}^{-} \backslash U_{1}$ from $\partial U_{1}$ to $\partial V_{1}$.

In the above construction, we may keep adding the disjoint intervals to our collection $\left\{\tau_{j}\right\}$ until it is true that each complementary interval of $\tau$ is contained in $V_{i}^{-}$for some $i$.

The argument may then be turned around to reach the following conclusion. Given $N<\infty$ there exists a compact set $K \subset \boldsymbol{H}$ such that each path $\tau$ from $z_{0}$ which contains at most $N$ disjoint intervals, where each projects to a path in some $V_{i}^{-} \backslash U_{i}$ between $\partial U_{i}$ and $\partial V_{i}$, lies in $K$. For such a $\tau$ is covered by at most $2 N+1$ components of $\left\{\pi^{-1}\left(V_{i}^{-}\right)\right\}$.

The next step in the proof of Lemma 2 is to make the assertion that there exists a number $d_{0}>0$ such that for any $\varphi \in Q(R)$, the $\varphi$-distance between $\partial U_{i}$ and $\partial V_{i}$ exceeds $d_{0}$, for all $i$. This is an immediate consequence of the compactness of $Q(R)$.

We now have enough information to prove the statements of Lemma 2. Argue by contradiction. If (i) is false there is a sequence of arcs $\tau_{n}$ from $z_{0}$ to $z_{n}$ with $\lim z_{n} \in \partial \boldsymbol{H}$ such that for some $\varphi_{n} \in Q(R)$ the $\varphi_{n}$-length of $\tau_{n}$ does not exceed $L$ for some $L<\infty$. In particular $\tau_{n}$ cannot contain more than $L / d_{0}$ disjoint intervals each of which is over some $V_{i}^{-} \backslash U_{i}$ as described above. But then there is some compact subset $K \subset \boldsymbol{H}$ containing all $\tau_{n}$, a contradiction.

Suppose (ii) is false. There is then a sequence of arcs $\tau_{n}$ from $z_{n}$ to $z_{n}^{\prime}$ where $\lim z_{n}=\zeta, \lim z_{n}^{\prime}=\zeta^{\prime}$ yet the $\varphi_{n}$-length of $\tau_{n}$ does not exceed some $L<\infty$, for some $\varphi_{n} \in Q(R)$. Again $\tau_{n}$ cannot contain more than $L / d_{0}$ disjoint intervals each over some $V_{i}^{-} \backslash U_{i}$ as described above. Therefore for each $n, \tau_{n}$ can be covered by $2 L / d_{0}+1$ components of $\left\{\pi\left(V_{i}^{-}\right)\right\}$. There exists a compact set $K$ such that for each $n$ there is a cover transformation $T_{n}$ such that $T_{n}\left(\tau_{n}\right) \subset K$. On the other hand each convergent subsequence of the Möbius transformations $\left\{T_{n}^{-1}\right\}$ converges uniformly on $K$ to a point on $\boldsymbol{H}$. This is a contradiction to the behavior of $\tau_{n}$. 
4. Corollary 1. (i) Let $K \subset \boldsymbol{H}$ be compact. The closure of the set of points which are at $\varphi^{*}$-distance $\leqq L<\infty$ from $K$ for some $\varphi \in Q(R)$ is compact.

(ii) For any pair of points $z_{0}, z \in \boldsymbol{H}$ and any $\varphi \in Q(R)$, there is a unique $\varphi^{*}$ geodesic in $\boldsymbol{H}$ between $z_{0}$ and $z$.

Proof: The set in (i) is closed by definition and by (i) of Lemma 2 it is bounded away from $\partial \boldsymbol{H}$. Part (ii) is a consequence of the completeness of the $\varphi$-metric; for details see [5].

Corollary 2 (Rigidity). Let $\tau\left(z, z^{\prime}\right)$ denote the $\varphi^{*}$-geodesic between $z$ and $z^{\prime}$. Then for any compact set $K \subset \boldsymbol{H}$, the number

$$
L=\sup \left|\tau\left(z, z^{\prime}\right)\right|_{\varphi^{*}}
$$

is finite, where the supremum of the $\varphi^{*}$-length $|\cdot|_{\varphi^{*}}$ is taken over all pairs of points $z, z^{\prime} \in K$ and all $\varphi \in Q(R)$. The number $L$ depends only on $K$.

Proof: Choose sequences $z_{n}, z_{n}^{\prime}, \varphi_{n}$ such that $\left|\tau_{n}\left(z_{n}, z_{n}^{\prime}\right)\right|_{\varphi_{n}} \rightarrow L$. By passing to subsequences we may assume that $z_{n} \rightarrow z \in K, z_{n}^{\prime} \rightarrow z^{\prime} \in K$, and $\varphi_{n} \rightarrow \varphi \in Q(R)$. Let $\tau\left(z, z^{\prime}\right)$ denote the $\varphi^{*}$-geodesic joining $z$ and $z^{\prime}$. Because of the uniform convergence

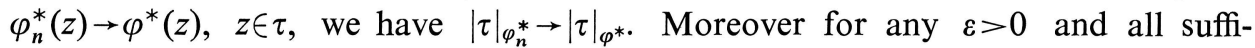
ciently large $n,\left|\tau_{n}\right| \varphi_{n}^{*} \leqq|\tau|_{\varphi_{n}^{*}}+\varepsilon$ because $\tau_{n}$ is the $\varphi_{n}^{*}$-geodesic and the pairs $\left(z, z_{n}\right)$, $\left(z^{\prime}, z_{n}^{\prime}\right)$ have uniformly short connections. The two relations lead to the inequality $L \leqq|\tau|_{\varphi^{*}}<\infty$.

5. Also very useful for our work in $\boldsymbol{H}$ is the following result.

Lemma 3. Let $\left\{z_{n}\right\},\left\{z_{n}^{\prime}\right\}$ be two sequences of points in $\boldsymbol{H}$ such that $\lim z_{n}=\zeta$, $\lim z_{n}^{\prime}=\zeta^{\prime}$ where $\zeta \neq \zeta^{\prime} \in \partial \boldsymbol{H}$. For given $\varphi \in Q(R)$, let $\tau_{n}$ be the $\varphi^{*}$-geodesic between $z_{n}$ and $z_{n}^{\prime}$. Then there exists $r<1$ such that for all $n$ and all $\varphi \in Q(R), \tau_{n} \cap\{z:|z| \leqq r\}$ $\neq \emptyset$.

Proof: Assume this is not the case for some $\varphi$. Then there is a subsequence $\left\{\tau_{m}\right\}$ tending uniformly to an interval $I$ on $\partial \boldsymbol{H}$ bounded by $\zeta, \zeta^{\prime}$. There exists a cover transformation $T$ over $R$ whose fixed points lie in the interior $I^{\circ}$. To find such a $T$ first recall that the fixed points of the elements of the universal covering group $G$ are dense on $\partial \boldsymbol{H}$. Choose $T_{1} \in G$ so that its attractive fixed point lies in $I^{\circ}$. Then choose $T_{2} \in G$ with different fixed points than $T_{1}$. For sufficiently large $k>0$, $T=T_{1}^{k} T_{2} T_{1}^{-k}$ fills the requirement.

Let $\sigma^{*}$ denote the hyperbolic line in $\boldsymbol{H}$ between the fixed points of $T$ (i.e. the axis of $T)$. Its projection $\sigma=\pi\left(\sigma^{*}\right)$ to $R$ is a closed, but not necessarily simple, curve. Let $\gamma$ be the $\varphi$-geodesic on $R$ in the free homotopy class of $\sigma$. It has a lift $\gamma^{*}$ with the same end points as $\sigma^{*}$. But the geodesic arcs $\left\{\tau_{m}\right\}$ are converging to the entire interval $I$ and therefore have geodesic subarcs $\tilde{\tau}_{m}$ connecting points of $\gamma^{*}$ for all large $m$. This contradicts the uniqueness of $\varphi^{*}$-geodesic connections. 
Because of the compactness of $Q(R)$, the argument remains valid if $\varphi$ varies in $Q(R)$.

6. The hyperbolic metric in $\boldsymbol{H}$ has the property that any two points of the closure $\overline{\boldsymbol{H}}$ can be joined by a uniquely determined geodesic. We are now going to prove the existence part of the analogous theorem for the $\varphi$-metric. The uniqueness part, which holds except for a special case mentioned below, has been shown in [3].

Theorem 1. Let $\varphi^{*}$ be the lift to $\boldsymbol{H}$ of a holomorphic quadratic differential $\varphi \not 0$ on a compact Riemann surface $R$. Then

(i) Every pair $z_{0} \in \boldsymbol{H}, \zeta \in \partial \boldsymbol{H}$ can be joined by a uniquely determined $\varphi^{*}$-geodesic ray $\gamma$.

(ii) Every pair of boundary points $\zeta, \zeta^{\prime} \neq \zeta$ of $\boldsymbol{H}$ can be joined by a $\varphi^{*}$-geodesic $\gamma$. It is uniquely determined except for the case of a lift of a closed $\vartheta$-trajectory of $\varphi$. In this case all the closed $\vartheta$-trajectories of the associated annulus, including the two boundary curves, have lifts connecting the same boundary points of $\boldsymbol{H}$.

Proof: (i) Let $z_{0} \in \boldsymbol{H}, \zeta \in \partial \boldsymbol{H}$ be given. Let $z_{n} \rightarrow \zeta$. We may of course assume that $\|\varphi\|=1$ and the $\varphi^{*}$-geodesic $\gamma_{n}$ connecting $z_{0}$ to $z_{n}$ has $\varphi^{*}$-length $L_{n}=n \cdot d$ for some fixed positive number $d$ (e.g. $d=1$ ). We parametrize it by means of the natural parameter $u$ with respect to the $\varphi^{*}$-metric. The representation of $\gamma_{n}$ is then given by a function $\gamma_{n}(u), 0 \leqq u \leqq L_{n}$. By the completeness lemma, the points $z_{n k}=\gamma_{n}(k \cdot d)$, $n \geqq k$, which are at a $\varphi^{*}$-distance $k \cdot d$ from $z_{0}$, are bounded away from $\partial \boldsymbol{H}$. We can therefore pick a subsequence of $\left\{z_{n}\right\}$ such that the sequence of points $z_{n 1}=\gamma_{n}(d)$ converges to a point $z_{01} \in \boldsymbol{H}$. From this we take a second subsequence such that the corresponding sequence of points $z_{n 2}=\gamma_{n}(2 d)$ converges to a point $z_{02} \in \boldsymbol{H}$, and so forth. Finally passing to the diagonal sequence, which we denote by $\left\{z_{n}\right\}$ again, we have arrived at a sequence of geodesics $\gamma_{n}$ of $\varphi$, connecting $z_{0}$ to $z_{n}$, of length $\left|\gamma_{n}\right|_{\varphi^{*}}=$ $L_{n} \rightarrow \infty$, such that the sequence of points $\left\{z_{n k}\right\}=\left\{\gamma_{n}(k d)\right\}$ converges for every $k: z_{n k} \rightarrow z_{0 k}, k=1,2, \ldots$. Let $\gamma$ be the curve composed of the geodesic connections $\gamma_{k}: z_{0, k-1} \ldots z_{0 k}, k=1,2, \ldots$ Then, clearly, the $\varphi^{*}$-length of every arc $\gamma_{k}$ is equal to $d$. Therefore, every subarc of $\gamma$ connecting $z_{0}$ to $z_{0 k}, k=1,2, \ldots$, is a geodesic. Thus, $\gamma$ is a geodesic ray, with initial point $z_{0}$, of infinite length. Because of Lemma 3 it tends to the boundary $\partial \boldsymbol{H}$, and from [3] we conclude that it has a well defined end point $\zeta^{\prime} \in \partial \boldsymbol{H}$. We claim $\zeta^{\prime}=\zeta$. If not, the geodesic connections $\gamma_{n}$ of $z_{0}$ and $z_{n}$ have subarcs tending uniformly to one of the subintervals, $I$, say, between $\zeta$ and $\zeta^{\prime}$. But this is a contradiction to Lemma 3.

(ii) Let $\zeta \neq \zeta^{\prime}$ and choose sequences of points $z_{n} \rightarrow \zeta, z_{n}^{\prime} \rightarrow \zeta^{\prime}$. Let $\gamma_{n}$ be the $\varphi^{*}$ geodesic joining $z_{n}$ to $z_{n}^{\prime}$. We know by Lemma 3 that there exists $r<1$ such that every $\gamma_{n}$ intersects the disk $|z| \leqq r$. We can therefore fix a point $z_{0 n} \in \gamma_{n},\left|z_{0 n}\right| \leqq r$, for each $n$. Using the above procedure for both half rays, with initial point $z_{0 n} \rightarrow z_{0}$, we find the desired $\varphi^{*}$-geodesic $\gamma$ which connects $\zeta$ and $\zeta^{\prime}$. 
7. We are now ready to prove the following result about convergence of sequences of geodesic rays on the universal covering surface.

Theorem 2. Suppose $\boldsymbol{R}$ is a compact Riemann surface and $\boldsymbol{H}$ is its universal covering surface realized as the unit disk. Suppose $\left\{\varphi_{n}\right\}$ is a sequence of normalized holomorphic quadratic differentials converging locally uniformly to a differential $\varphi$ on $R$ and $\left\{\varphi_{n}^{*}\right\}, \varphi^{*}$ denote the lifted differentials to $\boldsymbol{H}$. Let $\alpha^{*}$ be a regular trajectory ray of $\varphi^{*}$ with initial point $z_{0} \in \boldsymbol{H}$ and assume $z_{n} \rightarrow z_{0}$. Then any sequence of geodesic rays $\alpha_{n}^{*}$ of $\varphi_{n}^{*}$ with initial points $z_{n}$ and leaving $z_{n}$ with the limiting direction of $\alpha^{*}$ tends uniformly (with respect to the Euclidean metric in $\boldsymbol{H}$ ) to $\alpha^{*}$.

Proof: We first consider the case where the rays $\alpha_{n}^{*}$ are regular. We choose $0<r<1$ such that $z_{n} \in K=\{z:|z| \leqq r\}$ for all $n$. Given $L(K)$ according to Corollary 2 , let $\gamma^{*}$ be the subarc of $\alpha^{*}$ with initial point $z_{0}$ and $\varphi^{*}$-length $2 L(K)$. For all large enough values of $n$ the arc $\alpha_{n}^{*}$ has an initial arc $\gamma_{n}^{*}$ which is contained in an arbitrarily small rectangular neighbourhood (with respect to $\varphi^{*}$ ) of $\gamma^{*}$ and which has $\varphi_{n}^{*}$-length $>L(K)$. Therefore the arc $\alpha_{n}^{*} \backslash \gamma_{n}^{*}$ stays outside of $K$.

Assume now that the theorem is wrong. Then, because of the above remark, there is a closed, non-degenerate interval $I$ on $\partial \boldsymbol{H}$, and a subsequence of the differentials $\left\{\varphi_{n}^{*}\right\}$ and subintervals of the corresponding $\alpha_{n}^{*}$ which tend uniformly to $I$. But this is a contradiction to Lemma 3 .

Let now $\left\{\alpha_{n}^{*}\right\}$ be any geodesic rays. Choose a short $\varphi_{n}^{*}$-arc $\beta_{n}^{*}$ orthogonal to $\alpha_{n}^{*}$ through $z_{n}$ and on $\beta_{n}^{*}$, choose two points $z_{n}^{\prime}, z_{n}^{\prime \prime}$ on either side of $z_{n}$ with regular $\varphi_{n}^{*}$ trajectory rays $\alpha_{n}^{* \prime}$ and $\alpha_{n}^{* \prime \prime}$, respectively. Choose these to have the same initial direction as $\alpha_{n}^{*}$. Because of the divergence principle (see [5]) the rays $\alpha_{n}^{* \prime}$ and $\alpha_{n}^{*}$ cannot meet, nor can $\alpha_{n}^{* \prime \prime}$ and $\alpha_{n}^{*}$. The ray $\alpha_{n}^{*}$ therefore stays between the rays $\alpha_{n}^{* \prime}$ and $\alpha_{n}^{* \prime \prime}$. Since both sequences $\left\{\alpha_{n}^{* \prime}\right\}$ and $\left\{\alpha_{n}^{* \prime \prime}\right\}$ converge uniformly to $\alpha^{*},\left\{\alpha_{n}^{*}\right\}$ must as well.

8. A right extreme horizontal ray $\alpha^{*}$ of $\varphi^{*}$ - or equivalently $\alpha$ of $\varphi$ on $R$ - from a point $z_{0} \in \boldsymbol{H}$ is a geodesic ray composed of horizontal segments such that when travelling along $\alpha^{*}$, going from one segment to the next at a zero of $\varphi^{*}$, one takes the sharpest possible right turn. Similarly, left extreme horizontal rays are defined. And also one can speak of left and right extreme horizontal geodesics.

A right extreme horizontal geodesic $\alpha^{*}$ can be approximated from the right by regular $\varphi^{*}$-trajectories. Namely take any sequence $\left\{z_{n}\right\}$ which converges to $z \in \alpha^{*}$ from the right of $\alpha^{*}$ where a regular trajectory $\alpha_{n}^{*}$ passes through $z_{n}$. Then $\alpha_{n}^{*}$ converges to $\alpha^{*}$ uniformly in $\boldsymbol{H}$. Since $\alpha_{n}^{*}$ certainly converges to $\alpha^{*}$ uniformly on compact subsets of $\boldsymbol{H}$, the proof is a repetition of that of Theorem 2 . Similarly a left extreme $\alpha^{*}$ can be approximated from the left by regular trajectories.

Combining this approximation property of extreme geodesics with Theorem 2 yields the following extension. 
Theorem 2*. Hypotheses as in Theorem 2. Let $\alpha^{*}$ be a left or right extreme horizontal geodesic of $\varphi^{*}$. There exists a sequence of regular trajectories $\alpha_{n}^{*}$ of $\varphi_{n}^{*}$ such that $\alpha_{n}^{*}$ converges uniformly to $\alpha^{*}$ in $\boldsymbol{H}$.

9. For later work we will need the following lemma concerning differentials $\Psi \in Q(R)$. Its proof is an adaptation of the methods of [3].

Lemma 4. Let $\alpha_{1}, \ldots, \alpha_{n}$ be horizontal geodesics for $\Psi^{*}$ in $\boldsymbol{H}$ with the following property. The end points of the collection $\left\{\alpha_{i}\right\}$ are arranged in cyclic order $x_{1}, x_{2}, \ldots$, $x_{n+1}=x_{1}$ on $\partial \boldsymbol{H}$ where $x_{i}, x_{i+1}$ are the end points of $\alpha_{i}, 1 \leqq i \leqq n$. Assume no projection $\pi\left(\alpha_{i}\right)$ is a closed curve in $R$. Then to each complementary component $\Delta$ of $\cup \alpha_{i}$ in $\boldsymbol{H}$ corresponds some $j$ so that $\partial \Delta$ is the union of $\alpha_{j}$ and the interval $\left[x_{j}, x_{j+1}\right]$ that it faces on $\partial \boldsymbol{H}$.

Proof: Assume the statement is false and $\Delta$ is a complementary component whose boundary intersects $\partial \boldsymbol{H}$ at most at the points $\left\{x_{i}\right\}$. Suppose for example that $\partial \Delta$ contains a segment of $\alpha_{1}$ and fix a short vertical segment $\beta$ to $\alpha_{1}$ lying in $\Delta$. Consider the regular horizontal rays leaving one side $\beta^{+}$of $\beta$. They cannot cross any $\left\{\alpha_{i}\right\}$. Therefore each one is contained in $\Delta$ and terminates at one of the points $\left\{x_{i}\right\}$ on $\partial \boldsymbol{H}$. We claim that for sufficiently short $\beta$ all of them terminate at $x_{1}$ or all terminate at $x_{2}$. For as $\beta$ shrinks to its initial point on $\alpha_{1}$, the rays converge to a ray of $\alpha_{1}$. So we may assume all the regular rays from $\beta^{+}$terminate at $x_{1}$. Then after shortening $\beta$ again if necessary, all the regular rays from $\beta^{-}$terminate at $x_{2}$. We conclude that there are lots of regular trajectories with the same end points as $\alpha_{1}$, which is a contradiction.

10. Trees and stars. Consider the critical graph in $\boldsymbol{H}$ of some $\varphi^{*}, \varphi \in Q(R)$. A tree is a component of the critical graph. If $\alpha$ is a horizontal $\varphi^{*}$-geodesic, we denote by $\alpha^{\star}$ the tree that contains $\alpha$.

A tree is the union of zeros of $\varphi^{*}$, horizontal segments connecting zeros, and horizontal rays from a zero to a point on $\partial \boldsymbol{H}$. If the tree $\alpha^{*}$ is finite, that is contains a finite number of zeros of $\varphi^{*}$, we will refer to it as the star of $\alpha$.

Here is a list of properties of a tree $\alpha^{\star}$.

a) A tree $\alpha^{*}$ is the union of extreme $\varphi^{*}$-horizontal geodesics which are found as follows. Let $\zeta \in \alpha^{*}$ be a zero of order $n$. There are $(n+2)$ sectors determined at $\zeta$ by the horizontal rays. With respect to each of these, an extreme $\varphi^{*}$-horizontal geodesic $\varrho$ can be constructed. Orient $\varrho$ so that it is right extreme. Then $\alpha^{\star}$ appears to the left of $\varrho$. Make all possible such constructions and denote the resulting collection of extreme geodesics by $\left\{\alpha_{k}\right\}$.

b) Every extreme $\varphi^{*}$-horizontal geodesic contained in $\alpha^{\star}$ coincides with some $\alpha_{k}$.

c) If $\Delta$ is a component of $\boldsymbol{H \backslash \alpha}$ then $\Delta$ is simply connected and bounded by the union of some $\alpha_{k}$ and the interval of $\partial \boldsymbol{H}$ between its end points that its right side faces. 
d) If $\alpha_{1}$ is any $\varphi^{*}$-horizontal geodesic then either $\alpha_{1} \subset \alpha^{\star}$, or the two trees $\alpha_{1}^{\star}, \alpha^{\star}$ are disjoint. In the latter case $\alpha_{1}^{\star}$ lies in a complementary component $\Delta$ of $\alpha^{\star}$ and the end points of $\alpha_{1}$ do not separate the set of end points of all geodesics in $\alpha^{\star}$.

e) The tree $\alpha^{\star}$ is a star if and only if the decomposition $\left\{\alpha_{k}\right\}$ of (a) is finite. In this case there are a finite number of points arranged in positive cyclic order on $\partial \boldsymbol{H}, x_{1}, x_{2}, \ldots, x_{n+1}=x_{1}$, such that for suitable indexing, $\alpha_{i}$ has initial point $x_{i}$ and terminal point $x_{i+1}, 1 \leqq i \leqq n$.

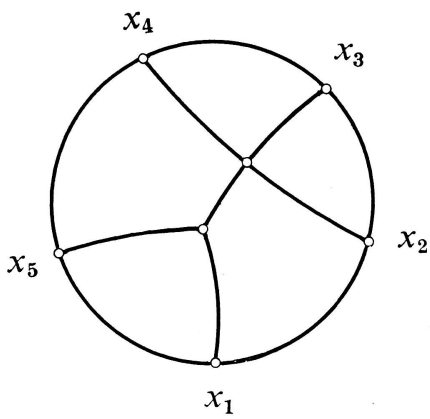

a star

11. For the remainder of our work we restrict our attention to a subset of admissible differentials $\varphi \in Q(R)$. The differential $\varphi$ is admissible if its critical horizontal graph in $R$ contains no simple loops. Equivalently, $\varphi$ is admissible if every tree for its lift $\varphi^{*}$ in $\boldsymbol{H}$ is finite, that is, a star.

We recall that the simple differential $\varphi[\gamma] \in Q(R)$ corresponding to the free homotopy class $[\gamma]$ of the simple loop $\gamma$ (not retractible to a point) is characterized by the property that all its regular trajectories are closed and lie in [ $\gamma]$. If $f: R \rightarrow S$ is a homeomorphism between surfaces, the correspondence $f_{\text {\# }}: \varphi[\gamma] \rightarrow \varphi[f(\gamma)]$ extends to a homeomorphism $f_{\text {\# }}: Q(R) \rightarrow Q(S)$ (see [2]). Given $\varphi \in Q(R)$ and its lift $\varphi^{*}$ to $\boldsymbol{H}, f_{\#}\left(\varphi^{*}\right)$ is well defined as the lift of $f_{\#}(\varphi)$.

The homeomorphism $f: R \rightarrow S$ also determines a homeomorphism $f^{*}: \partial \boldsymbol{H} \rightarrow$ $\partial \boldsymbol{H}$ (see Section 1). It has the property that $p, q \in \partial \boldsymbol{H}$ are the fixed points of a cover transformation over $R$ if and only if $f^{*}(p), f^{*}(q)$ are the fixed points of one over $S$.

Theorem 3. Suppose $f: R \rightarrow S$ is a homeomorphism between compact surfaces and $\varphi \in Q(R)$ is admissible. Then $f_{\#}(\varphi) \in Q(S)$ is also admissible. Furthermore if $\zeta_{1}, \zeta_{2} \in \partial \boldsymbol{H}$ are the end points of a horizontal trajectory (extreme horizontal geodesic) $\alpha$ of the lift $\varphi^{*}$ of $\varphi$ to $\boldsymbol{H}$, then $f^{*}\left(\zeta_{1}\right), f^{*}\left(\zeta_{2}\right)$ are the end points of a unique horizontal trajectory (respectively, extreme horizontal geodesic) of $f_{\#}\left(\varphi^{*}\right)$. If $\alpha \star$ is the star of an extreme horizontal geodesic $\alpha$ of $\varphi^{*}$ and $\left[x_{1}, \ldots, x_{n+1}=x_{1}\right]$ are its end points on $\partial \boldsymbol{H}$, then $\left[f^{*}\left(x_{1}\right), \ldots, f^{*}\left(x_{n+1}\right)=f^{*}\left(x_{1}\right)\right]$ are the points on $\partial \boldsymbol{H}$ for the star of the corresponding extreme geodesic of $f_{\#}\left(\varphi^{*}\right)$. 
12. Proof: Step 1. For some sequence of simple differentials, $\varphi=\lim \varphi\left[\gamma_{n}\right]$, fix a horizontal or (right or left) extreme geodesic $\alpha$ for the lift $\varphi^{*}$ of $\varphi$ in $\boldsymbol{H}$. Theorem $2^{*}$ shows the existence of a horizontal trajectory $\alpha_{n}$ of $\varphi\left[\gamma_{n}\right]^{*}$ such that $\lim \alpha_{n}=\alpha$, uniformly in $\boldsymbol{H}$. In particular, the end points $p_{n}, q_{n}$ of $\alpha_{n}$ on $\partial \boldsymbol{H}$ converge to those $p, q$ of $\alpha$. The end points $p_{n}, q_{n}$ are the fixed points of a cover transformation $T_{n}$ such that $T_{n}\left(\alpha_{n}\right)=\alpha_{n}$. The projection $\pi\left(\alpha_{n}\right)$ in $R$ is a closed trajectory of $\varphi\left[\gamma_{n}\right]$ necessarily freely homotopic to $\gamma_{n}$. In fact the trajectories of $\varphi\left[\gamma_{n}\right]$ sweep out an annular region $A_{n}$. The component $A_{n}^{*}$ of $\left\{\pi^{-1}\left(A_{n}\right)\right\}$ in $\boldsymbol{H}$ which contains $\alpha_{n}$ converges to $\alpha$ uniformly in $\boldsymbol{H}$; the relative boundary of $A_{n}^{*}$ in $\boldsymbol{H}$ is the union of a right extreme and a left extreme horizontal geodesic of $\varphi\left[\gamma_{n}\right]$.

Correspondingly over $S$ there is a family of horizontal trajectories of $\varphi\left[f\left(\gamma_{n}\right)\right]^{*}$ all of which share the end points $f^{*}\left(p_{n}\right), f^{*}\left(q_{n}\right)$. Fix one of these and denote it $\beta_{n}$. Since the end points of $\beta_{n}$ converge to $f^{*}(p), f^{*}(q)$ we claim that $\left\{\beta_{n}\right\}$ itself converges to a horizontal geodesic of $f_{\#}\left(\varphi^{*}\right)=\lim \varphi\left[f\left(\gamma_{n}\right)\right]^{*}$. (At this point, we do not also claim that $\beta$ is extreme.)

But this is an easy consequence of Theorem 1 . Note that $f^{*}(p)$ and $f^{*}(q)$ are not fixed points of a cover transformation over $S$ since $p, q$ are not the fixed points of one over $R$. Therefore there can be at most one $f_{\#}\left(\varphi^{*}\right)$-geodesic with end points $f^{*}(p), f^{*}(q)$. By the Existence Theorem 1 there is such a geodesic $\beta$. The proof of this theorem can be repeated for the geodesics $\beta_{n}$ : from $\varphi\left[\gamma_{n}\right]^{*} \rightarrow \varphi^{*}$ locally uniformly and $f^{*}\left(p_{n}\right) \rightarrow f^{*}(p), f^{*}\left(q_{n}\right) \rightarrow f^{*}(q)$ we conclude that $\beta_{n} \rightarrow \beta$ uniformly on $\boldsymbol{H}$. Evidently $\beta$ must be horizontal.

Step 2. If $\alpha$ is not a trajectory, its star $\alpha \star$ is the union of a finite number of extreme $\varphi^{*}$-horizontal geodesics $\alpha_{1}, \alpha_{2}, \ldots, \alpha_{n}$ with consecutive end points $\left(x_{1}, x_{2}\right)$, $\left(x_{2}, x_{3}\right), \ldots,\left(x_{n}, x_{1}\right)$ on $\partial \boldsymbol{H}$. Over $S$ we have constructed the $f_{\#}\left(\varphi^{*}\right)$-horizontal geodesics $\beta_{1}, \ldots, \beta_{n}$ which have the corresponding end points $\left(f^{*}\left(x_{1}\right), f^{*}\left(x_{2}\right)\right), \ldots$, $\left(f^{*}\left(x_{n}\right), f^{*}\left(x_{1}\right)\right)$ on $\partial \boldsymbol{H}$.

Moreover, by Lemma 4 , each complementary component $\Delta$ of $\cup \beta_{i}$ in $\boldsymbol{H}$ is bounded by some $\beta_{j}$ and the interval $\left[f^{*}\left(x_{j}\right), f^{*}\left(x_{j+1}\right)\right]$ that it faces on $\partial \boldsymbol{H}$.

Step 3. Conversely, suppose that $\beta_{0}$ is a trajectory or an extreme (left or right) $f_{\#}\left(\varphi^{*}\right)$-horizontal geodesic. By considering $f^{-1}$ and $\left(f^{*}\right)^{-1}$ there is a corresponding $\varphi^{*}$-horizontal geodesic $\alpha_{0}$. The argument of Step 1 could be repeated to verify this except for the fact that we do not yet know that $\left(f^{*}\right)^{-1}(p),\left(f^{*}\right)^{-1}(q)$, where $p, q$ are the end points of $\beta_{0}$, are not the fixed points of a cover transformation over $R$ and therefore we do not know there is a unique $\varphi^{*}$-geodesic ketween them. However this difficulty is easy to overcome by using instead the fact that there is a unique $\varphi^{*}$ geodesic from a given point $z \in \boldsymbol{H}$ to a prescriked $\zeta \in \partial \boldsymbol{H}$. Given a sequence of trajectories $\gamma_{n}$ of some $\varphi_{n}^{*} \rightarrow \varphi$ where the end points $\zeta_{n}, \zeta_{n}^{\prime}$ of $\gamma_{n}$ are known to converge to points $\zeta \neq \zeta^{\prime} \in \boldsymbol{H}$ we can choose $z_{n} \in \gamma_{n}$ so that for a subsequence, $\lim z_{m}=$ $z \in \boldsymbol{H}$. The ray in $\gamma_{m}$ from $z_{m}$ to $\zeta_{m}$ (or to $\zeta_{m}^{\prime}$ ) converges to the geodesic ray from $z$ to $\zeta$ (or to $\zeta^{\prime}$ ). As in Step 1, we end up with a $\varphi^{*}$-horizontal geodesic $\alpha_{0}$ that corresponds to $\beta_{0}$. 
We claim that $\alpha_{0}$ is either a trajectory or an extreme horizontal geodesic, depending on what $\beta_{0}$ is.

To understand why this is so consider its star $\alpha_{0}^{\star}$ which is formed by extreme horizontal geodesics $\alpha_{1}, \ldots, \alpha_{n}$. If $\alpha_{0}$ is not itself extreme then its end points are some $x_{i}$ and $x_{j}$ with $j \neq i \pm 1$ (notation as in Step 2 for $\alpha$ ). Back over $S$ there are a finite number of $f_{\#}\left(\varphi^{*}\right)$-horizontal geodesics $\beta_{1}, \ldots, \beta_{n}$ as descriked in Step 2. The original $\beta_{0}$ lies in their union $\cup \beta_{i}$ since its end points do (Lemma 4). But its end points $f^{*}\left(x_{i}\right)$, $f^{*}\left(x_{j}\right)$ are not consecutive points on $\partial \boldsymbol{H}$ with respect to $f^{*}\left(x_{1}\right), f^{*}\left(x_{2}\right), \ldots$. Therefore $\beta_{0}$ cannot be extreme, since it can be viewed as keing formed from maximal noncritical segments of the collection $\left\{\beta_{i}\right\}$. This is a contradiction.

The same argument shows that, for example, $\beta_{0}$ is actually a trajectory if and only if $\alpha_{0}$ is also one.

Step 4. Now given $\alpha$ as in Step 1 return to examine more closely the corresponding $f_{\#}\left(\varphi^{*}\right)$-horizontal geodesic $\beta$. Consider its tree $\beta \star$ and the associated decomposition $\left\{\beta_{k}\right\}$ into right extreme geodesics. By Step 3, to each $\beta_{k}$ corresponds an extreme $\varphi^{*}$-horizontal geodesic $\alpha_{k}$ over $R$. The orientation of $\beta_{k}$ induces via its end points an orientation of $\alpha_{k}$. Since $f^{*}$ preserves orientation $\alpha_{k}$ will also be right extreme with respect to $\varphi^{*}$.

Now we analyze the collection $\left\{\alpha_{k}\right\}$ of extreme $\varphi^{*}$-horizontal geodesics over $R$. Lying to the right of each $\alpha_{k} \beta \star$ is a complementary component of $\cup \alpha_{k}$. We claim that there are no other complementary components of $\cup \alpha_{k}$ in $\boldsymbol{H}$.

Suppose that we are wrong and a complementary component $\Delta$ lies to the left of every $\alpha_{k}$. Since for each $k$, the projection $\pi\left(\alpha_{k}\right)$ is a dense geodesic in $R$, there is a point $\zeta$ in the interior of $\Delta$ such that for some cover transformation $T$ over $R$, $T \zeta \in \alpha_{k}$. Then $T^{-1} \alpha_{k}$ is an extreme geodesic through $\zeta$ which necessarily separates the collection $\left\{\alpha_{k}\right\}$. Therefore by property (d) in Section 10 above, the $f_{\#}\left(\varphi^{*}\right)$-geodesic $\sigma$ corresponding to $T^{-1} \alpha_{k}$ lies in $\beta^{\star}$. But since $T\left(T^{-1} \alpha_{k}\right)=\alpha_{k}$, so also $\left(f^{*} T f^{*-1}\right) \sigma=\beta_{k}$ since this is true of the end points on $\partial \boldsymbol{H}$. But then $\sigma$ is necessarily extreme and by property (b) is a member of the collection $\left\{\beta_{k}\right\}$. This contradicts our choice of $T^{-1} \alpha_{k}$ in the interior of $\Delta$.

We conclude that $\cup \alpha_{k}$ is a closed connected set in $\boldsymbol{H}$. Since each tree for $\varphi^{*}$ is finite, there can only be a finite numker of the $\left\{\alpha_{k}\right\}$. Furthermore, $\cup \alpha_{k}$ contains $\alpha$ since the end points of $\alpha$ on $\partial \boldsymbol{H}$ lie to the left of all $\left\{\alpha_{k}\right\}$ (the end points of $\beta$ lie to the left of all $\left.\left\{\beta_{k}\right\}\right)$.

Consequently $\cup \alpha_{k}$ can only be the star $\alpha^{\star}$ of $\alpha$. We have already noted that there are only a finite number of the extreme geodesics $\left\{\alpha_{k}\right\}$ and one of these must be $\alpha$ itself. Over $S$, there are only a finite number (the same number) of $\left\{\beta_{k}\right\}$ one of which must be $\beta$. In fact $\cup \beta_{k}$ is $\beta^{\star}$. The proof of Theorem 3 is now complete.

13. We will apply Theorem 3 to the simplest case, that on the compact surface $R, \varphi \in Q(R)$ satisfies 
(i) Each zero of $\varphi$ is a simple zero.

(ii) There is no $\varphi$-horizontal segment connecting two zeros.

Note that if $\varphi d w^{2}$ satisfies (i) then except for at most a countable number of values of $\vartheta, e^{i \vartheta} \varphi d w^{2}$ also satisfies (ii).

For such a differential, Theorem 3 can be refined as follows. As before, $f: R \rightarrow S$ is a homeomorphism onto another (or the same) surface $S$.

Theorem 4. Assume $\varphi d w^{2}$ on $R$ satisfies (i) and (ii). Then $f_{\#}(\varphi)$ also satisfies (i) and (ii) and there is a natural one-to-one correspondence between the horizontal trajectories and the $(4 g-4)$ zeros of $\varphi$ with respect to $R$, and those of $f_{\#}(\varphi)$ with respect to $S$. The correspondence is continuous in the Teichmüller metric.

Proof. In this case each zero $\xi$ of $\varphi^{*}$ in $\boldsymbol{H}$ gives rise to a star consisting of three critical rays from $\xi$. The first statement of Theorem 4 is therefore the specialization of Theorem 3.

As $(S, f) \rightarrow(R$, id $)$ in the Teichmüller metric it follows from the heights theorem [2] that up in $\boldsymbol{H}, f_{\text {\# }}\left(\varphi^{*}\right)$ converges locally uniformly to $\varphi^{*}$.

Theorem 2 shows that each trajectory of $\varphi^{*}$ is the limit, uniformly in $\boldsymbol{H}$, of the corresponding trajectory of $f_{\#}\left(\varphi^{*}\right)$. Since the horizontal geodesics of $\varphi^{*}$ are automatically extreme we can also assert that more generally, each horizontal geodesic of $\varphi^{*}$ is the limit, uniformly in $\boldsymbol{H}$, of the corresponding one of $f_{\#}\left(\varphi^{*}\right)$. The same reasoning applies to any other convergent sequence in Teichmüller space.

\section{References}

[1] Ahlfors, L. V.: On quasiconformal mappings. - J. Analyse Math. 3, 1954, 1-58.

[2] Marden, A., and K. Strebel: The heights theorem for quadratic differentials on Riemann surfaces. - Acta Math. 153, 1984, 153-211.

[3] Marden, A., and K. Strebel: On the ends of trajectories. - To appear in Differential geometry and complex analysis, H. E. Rauch memorial volume. Springer Verlag, Berlin, 1985.

[4] MAsur, H.: The Jenkins-Stretel differentials with one cylinder are dense. - Comment. Math. Helv. 54, 1979, 179-184.

[5] Strebel, K.: Quadratic Differentials. - Ergebnisse der Mathematik und ihrer Grenzgebiete (3) 5. Springer-Verlag, Berlin-Heidelberg-New York-Tokyo, 1984.

University of Minnesota

School of Mathematics

Minneapolis, Minnesota 55455

USA

and

University of Warwick

Department of Mathematics

Coventry

England

Received 7 May 1984
University of Zürich

Department of Mathematics

CH-8001 Zürich

Switzerland 